\title{
Conformations of $p$-tert-butylcalix[8]arene in solvated crystal structures
}

\author{
Aleksandra Kieliszek and Maura Malinska* \\ Faculty of Chemistry, University of Warsaw, Poland \\ E-mail: mmalinska@chem.uw.edu.pl \\ Phone: +48225526356
}

\section{List of Figures}

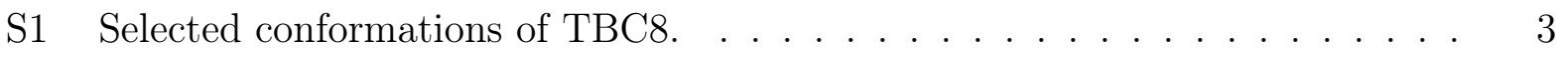

S2 Labelling of molecular structure of $\mathrm{TBC} 8$ in $1 \ldots \ldots \ldots 6$

S3 Labelling of molecular structure of $\mathrm{TBC} 8$ in $2 \ldots \ldots \ldots 7$

S4 Labelling of molecular structure of $\mathrm{TBC} 8$ in $3 \ldots \ldots \ldots$

S5 Labelling of molecular structure of $\mathrm{TBC} 8$ in $4 \ldots \ldots \ldots$

S6 Labelling of molecular structure of $\mathrm{TBC} 8$ in $5 \ldots \ldots \ldots$

S7 Labelling of molecular structure of TBC8 in $6 \ldots \ldots \ldots 11$

S8 Labelling of molecular structure of TBC8 in 7 . . . . . . . . . . . . . 12

S9 Photographic image of crystallization experiment of TBC8 in DMSO. Only prismatic crystal XRD data were collected. . . . . . . . . . . . . 13

\section{List of Tables}

S1 XRD data collection for $1-4 \ldots \ldots \ldots \ldots \ldots \ldots \ldots \ldots$ 
S2 XRD data collection for $5-7 \ldots \ldots \ldots \ldots$

S3 Hydrogen bonds for chloroform@TBC8 (1). ${ }^{1} 1-\mathrm{X}, 2-\mathrm{Y}, 1-\mathrm{Z},{ }^{2} 1-\mathrm{X}, 1-\mathrm{Y}, 2-\mathrm{Z}$. . . 6

S4 Hydrogen bonds for DMF@TBC8 (2) . . . . . . . . . . . . . . . 7

S5 Hydrogen bonds for DMF@TBC8 (3) . . . . . . . . . . . . . . 8

S6 Hydrogen bonds for ethyl acetate@TBC8 (4) . . . . . . . . . . . . . 9

S7 Hydrogen bonds for acetonitrile@TBC8 (5) . . . . . . . . . . . . . 10

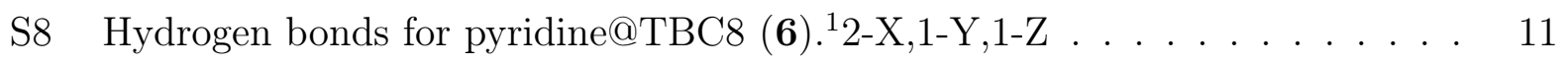

S9 Hydrogen bonds for DMSO@TBC8 (7). ${ }^{1} 1-\mathrm{X}, 1-\mathrm{Y}, 1-\mathrm{Z} \ldots \ldots . . . . . . . \quad 12$ 

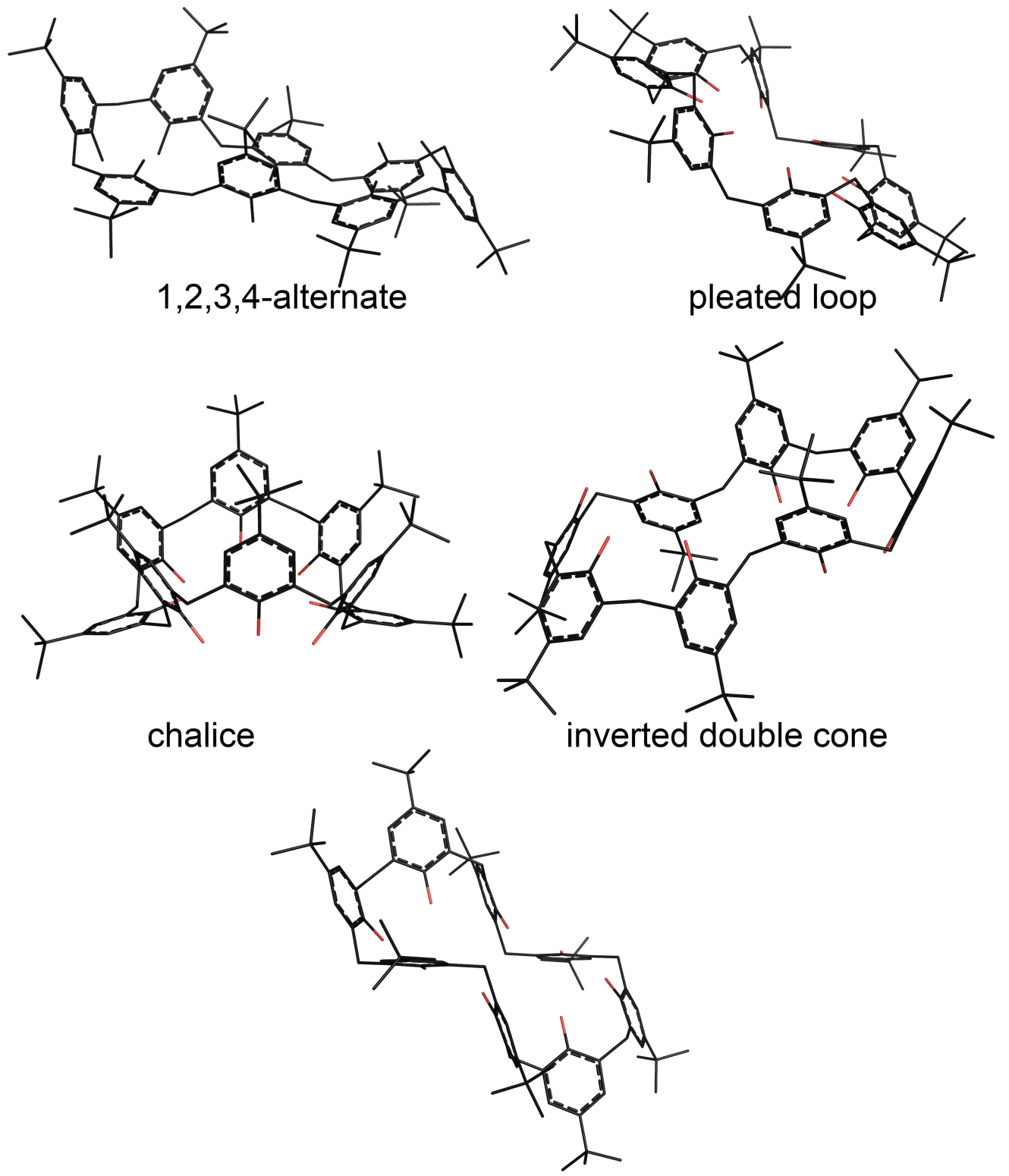

chair

Figure S1: Selected conformations of TBC8. 
Table S1: XRD data collection for $\mathbf{1 - 4}$

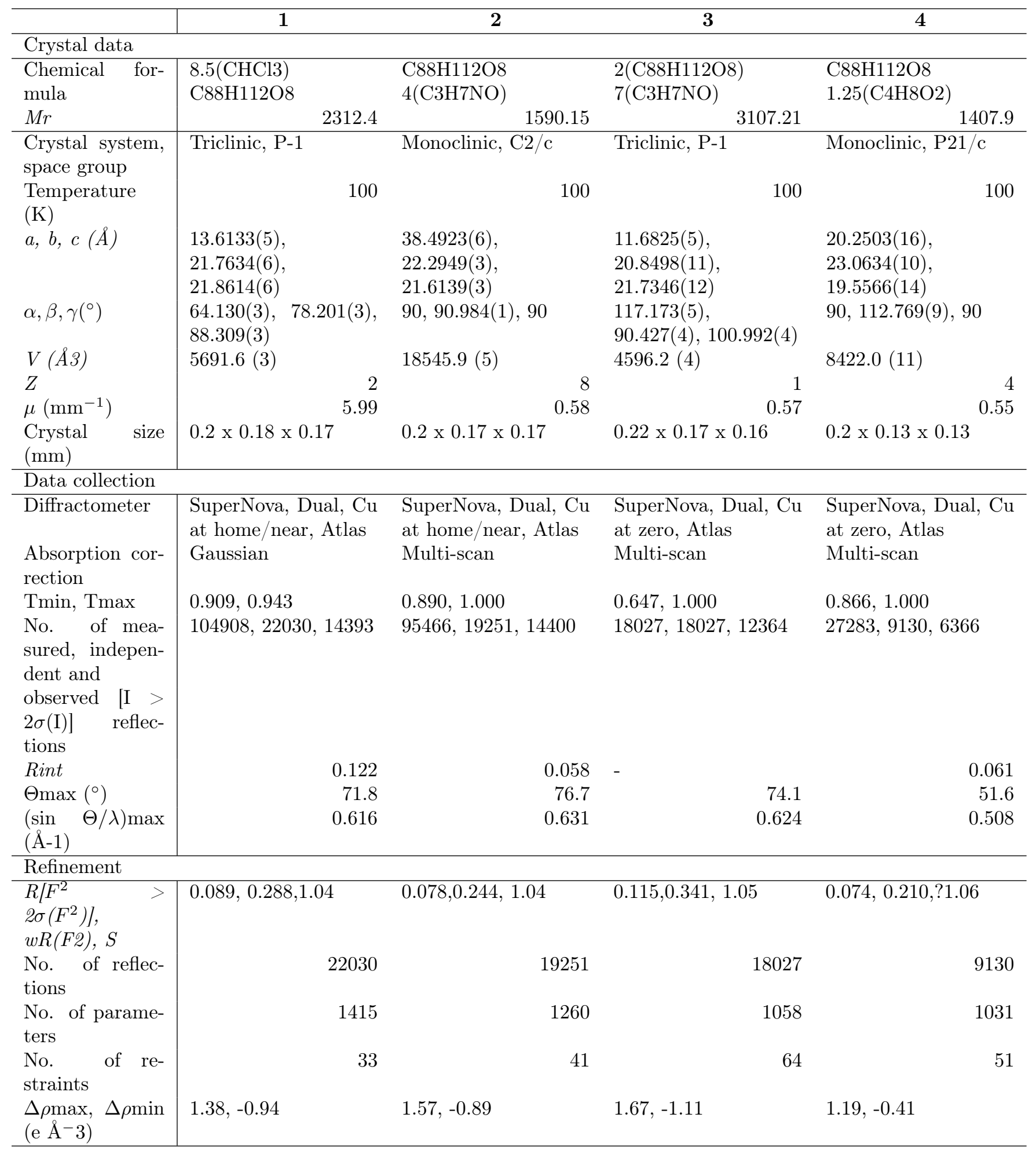


Table S2: XRD data collection for $\mathbf{5 - 7}$

\begin{tabular}{|c|c|c|c|}
\hline & 5 & 6 & 7 \\
\hline \multicolumn{4}{|l|}{ Crystal data } \\
\hline $\begin{array}{l}\text { Chemical for- } \\
\text { mula }\end{array}$ & $\mathrm{C} 88 \mathrm{H} 112 \mathrm{O} 8 \mathrm{C} 2 \mathrm{H} 3 \mathrm{~N}$ & $\begin{array}{l}\mathrm{C} 88 \mathrm{H} 112 \mathrm{O} 8 \\
8(\mathrm{C} 5 \mathrm{H} 5 \mathrm{~N})\end{array}$ & $\begin{array}{l}3(\mathrm{C} 2 \mathrm{H} 6 \mathrm{OS}) \\
\mathrm{C} 88 \mathrm{H} 112 \mathrm{O} 8\end{array}$ \\
\hline $\mathrm{Mr}$ & 1338.82 & 1930.57 & 1532.15 \\
\hline $\begin{array}{l}\text { Crystal system, } \\
\text { space group }\end{array}$ & Monoclinic, P21/c & Triclinic, P-1 & Triclinic, P-1 \\
\hline $\begin{array}{l}\text { Temperature } \\
\text { (K) }\end{array}$ & 100 & 100 & 100 \\
\hline $\mathrm{a}, \mathrm{b}, \mathrm{c}(\AA)$ & $\begin{array}{l}20.131(2) \\
22.9266(14) \\
19.7610(18)\end{array}$ & $\begin{array}{l}10.0590 \\
16.7828(6) \\
18.1772(5)\end{array}$ & $\begin{array}{l}\text { 13.2964(11), } \\
18.0257(15), \\
19.9051(16)\end{array}$ \\
\hline$\alpha, \beta, \gamma\left(^{\circ}\right)$ & $90,112.493(10), 90$ & $\begin{array}{l}113.405(3), \\
93.244(2), 94.224(2)\end{array}$ & $\begin{array}{l}93.456(7) \\
105.596(7) \\
104.113(7)\end{array}$ \\
\hline V $(\AA 3)$ & $8426.7(14)$ & $2796.12(15)$ & $4415.8(7)$ \\
\hline & 4 & 1 & 2 \\
\hline$\mu \mathrm{mm}-1)$ & 0.51 & 0.55 & 1.21 \\
\hline $\begin{array}{l}\text { Crystal size } \\
(\mathrm{mm})\end{array}$ & $0.2 \times 0.17 \times 0.15$ & $0.3 \times 0.2 \times 0.1$ & $0.18 \times 0.14 \times 0.12$ \\
\hline \multicolumn{4}{|l|}{ Data collection } \\
\hline Diffractometer & $\begin{array}{l}\text { SuperNova, Dual, Cu } \\
\text { at zero, Atlas }\end{array}$ & $\begin{array}{l}\text { SuperNova, Dual, } \mathrm{Cu} \\
\text { at zero, Atlas }\end{array}$ & $\begin{array}{l}\text { SuperNova, Dual, Cu } \\
\text { at home/near, Atlas }\end{array}$ \\
\hline Tmin, Tmax & $0.463,1.000$ & $0.905,1.000$ & $0.815,1.000$ \\
\hline $\begin{array}{l}\text { No. of mea- } \\
\text { sured, inde- } \\
\text { pendent and } \\
\text { observed [I > } \\
2 \sigma \mathrm{I}) \text { ] reflections }\end{array}$ & $10084,5450,3971$ & $45337,11436,9275$ & $11821,6142,4613$ \\
\hline Rint & 0.077 & 0.034 & 0.046 \\
\hline$\Theta \max \left({ }^{\circ}\right)$ & 44.3 & 74.5 & 42.6 \\
\hline$\left(\sin _{(\AA-1)} \Theta / \lambda\right) \max$ & 0.453 & 0.625 & 0.439 \\
\hline \multicolumn{4}{|l|}{ Refinement } \\
\hline $\begin{array}{l}\mathrm{R}[\mathrm{F} 2>2 \sigma(\mathrm{F} 2)], \\
\mathrm{wR}(\mathrm{F} 2), \mathrm{S}\end{array}$ & $0.092,0.285,1.02$ & $0.049,0.131,1.02$ & $0.090,0.266,1.04$ \\
\hline $\begin{array}{l}\text { No. of reflec- } \\
\text { tions }\end{array}$ & 5450 & 11436 & 6142 \\
\hline $\begin{array}{l}\text { No. of parame- } \\
\text { ters }\end{array}$ & 670 & 703 & 1011 \\
\hline $\begin{array}{l}\text { No. of re- } \\
\text { straints }\end{array}$ & 111 & 0 & 18 \\
\hline $\begin{array}{l}\Delta \rho \max , \Delta \rho \min \\
(\mathrm{e} \AA-3)\end{array}$ & $0.79,-0.32$ & $0.64,-0.52$ & $1.47,-0.53$ \\
\hline
\end{tabular}




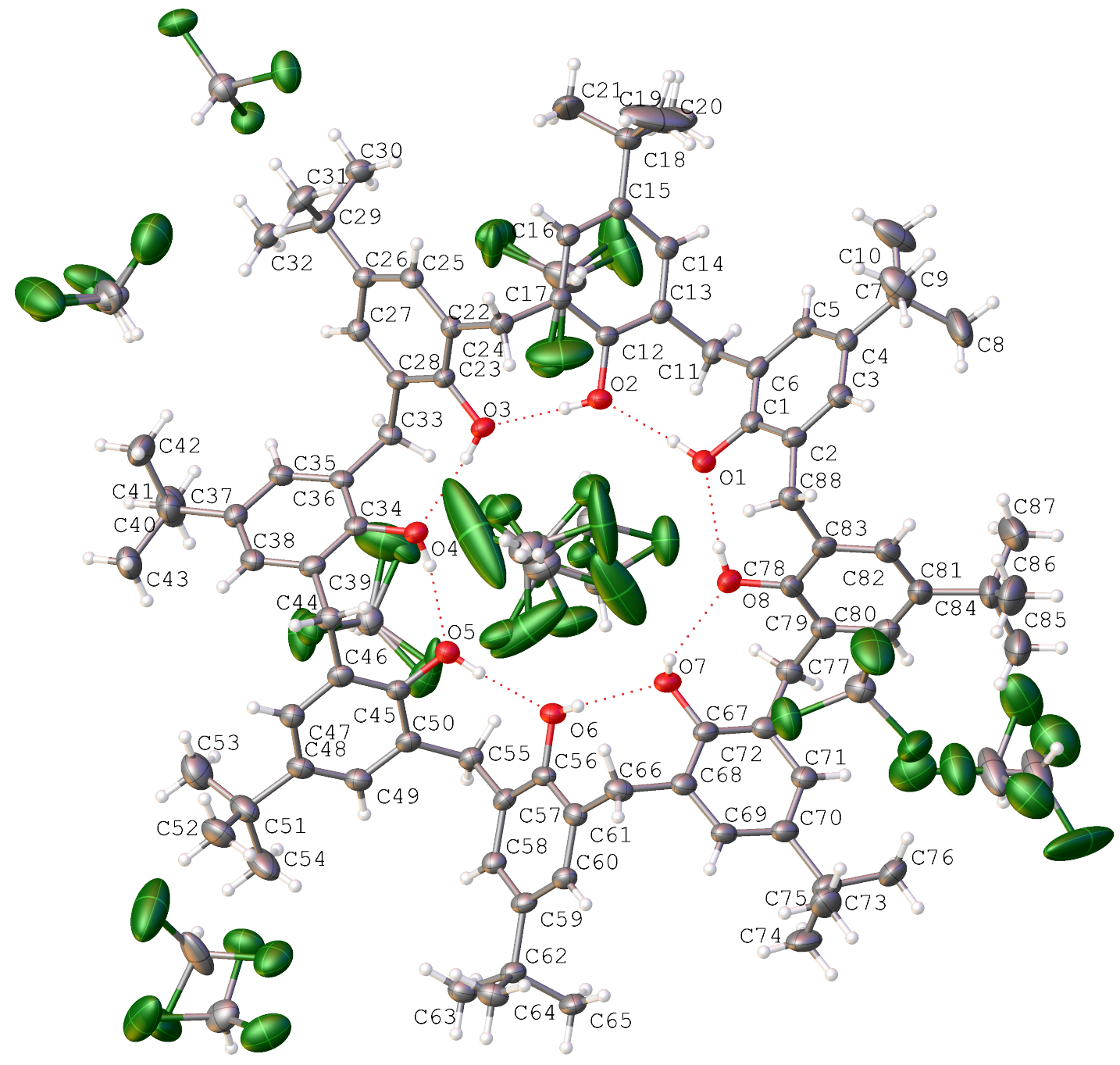

Figure S2: Labelling of molecular structure of TBC8 in $\mathbf{1 .}$

Table S3: Hydrogen bonds for chloroform@TBC8 (1). ${ }^{1} 1-\mathrm{X}, 2-\mathrm{Y}, 1-\mathrm{Z},{ }^{2} 1-\mathrm{X}, 1-\mathrm{Y}, 2-\mathrm{Z}$

\begin{tabular}{lllll}
\hline $\mathrm{D}$ & $\mathrm{H}$ & $\mathrm{A}$ & $\mathrm{d}(\mathrm{D}-\mathrm{A}) / \AA$ & $\mathrm{D}-\mathrm{H}-\mathrm{A} /{ }^{\circ}$ \\
\hline $\mathrm{O} 1$ & $\mathrm{H} 1$ & $\mathrm{O} 2$ & $2.696(4)$ & 169.4 \\
$\mathrm{O} 2$ & $\mathrm{H} 2$ & $\mathrm{O} 3$ & $2.740(4)$ & 176.3 \\
$\mathrm{O} 3$ & $\mathrm{H} 3$ & $\mathrm{O} 4$ & $2.724(4)$ & 152.8 \\
$\mathrm{O} 4$ & $\mathrm{H} 4$ & $\mathrm{O} 5$ & $2.706(4)$ & 156.8 \\
$\mathrm{O} 5$ & $\mathrm{H} 5$ & $\mathrm{O} 6$ & $2.702(4)$ & 174.2 \\
$\mathrm{O} 6$ & $\mathrm{H} 6$ & $\mathrm{O} 7$ & $2.660(5)$ & 173.9 \\
$\mathrm{O} 7$ & $\mathrm{H} 7$ & $\mathrm{O} 8$ & $2.674(4)$ & 178.4 \\
$\mathrm{O} 7$ & $\mathrm{H} 7$ & $\mathrm{Cl} 45^{1}$ & $3.195(14)$ & 102.8 \\
$\mathrm{O} 8$ & $\mathrm{H} 8$ & $\mathrm{O}^{2}$ & $2.687(4)$ & 143.4 \\
$\mathrm{C} 98$ & $\mathrm{H} 98$ & $\mathrm{Cl1}^{2}$ & $3.691(14)$ & 135.5 \\
$\mathrm{C} 97$ & $\mathrm{H} 97$ & $\mathrm{Cl1}^{2}$ & $3.73(3)$ & 137.5 \\
\hline
\end{tabular}




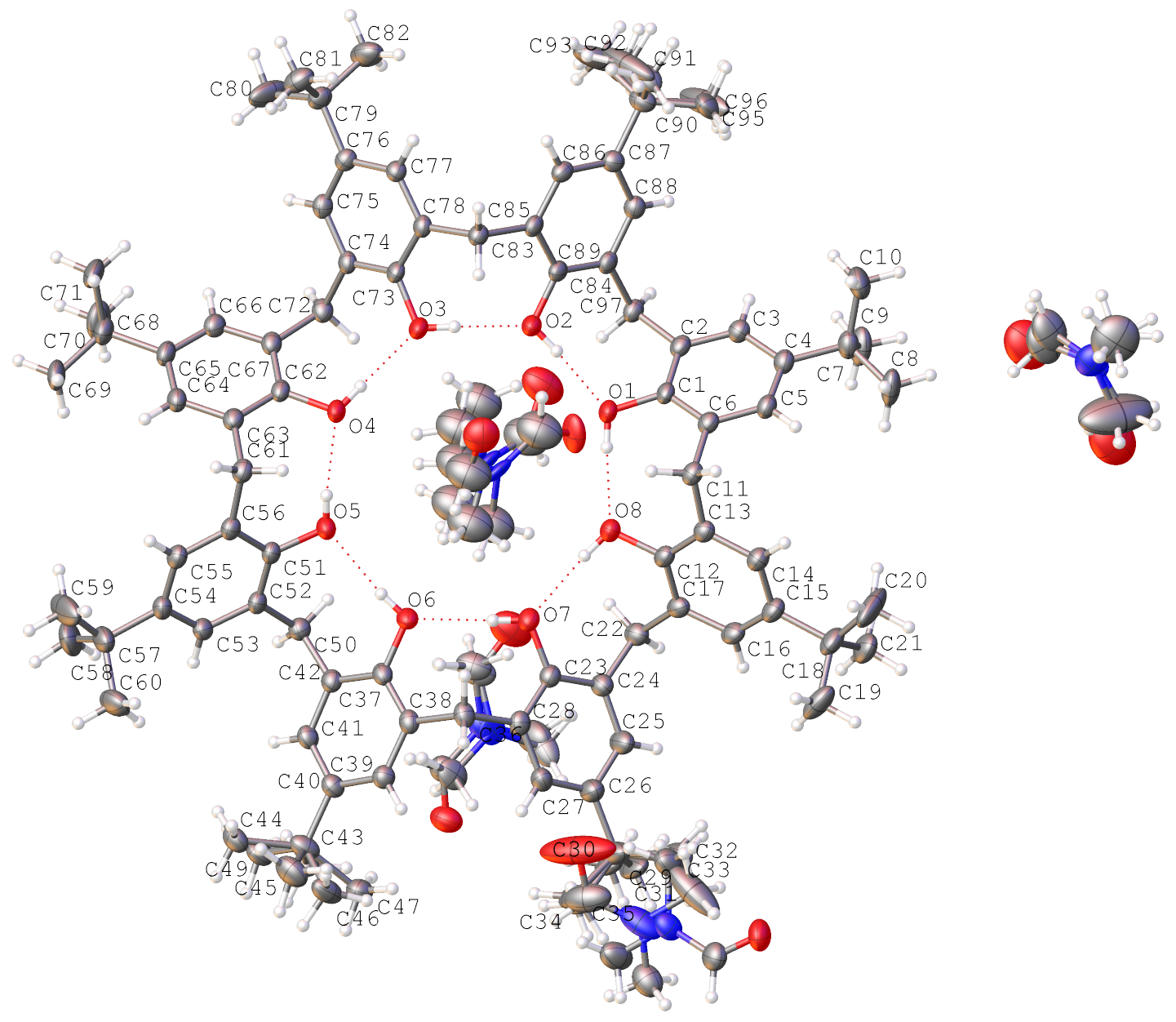

Figure S3: Labelling of molecular structure of TBC8 in 2.

Table S4: Hydrogen bonds for DMF@TBC8 (2)

\begin{tabular}{lllll}
\hline $\mathrm{D}$ & $\mathrm{H}$ & $\mathrm{A}$ & $\mathrm{d}(\mathrm{D}-\mathrm{A}) / \AA$ & $\mathrm{D}-\mathrm{H}-\mathrm{A} /{ }^{\circ}$ \\
\hline $\mathrm{O} 1$ & $\mathrm{H} 1$ & $\mathrm{O} 8$ & $2.725(3)$ & 174.7 \\
$\mathrm{O} 2$ & $\mathrm{H} 2$ & $\mathrm{O} 1$ & $2.621(3)$ & 171.4 \\
$\mathrm{O} 3$ & $\mathrm{H} 3$ & $\mathrm{O} 2$ & $2.588(3)$ & 173.5 \\
$\mathrm{O} 4$ & $\mathrm{H} 4$ & $\mathrm{O} 3$ & $2.662(3)$ & 155.6 \\
$\mathrm{O} 5$ & $\mathrm{H} 5$ & $\mathrm{O} 4$ & $2.718(3)$ & 158.3 \\
$\mathrm{O} 6$ & $\mathrm{H} 6$ & $\mathrm{O} 5$ & $2.775(3)$ & 172.8 \\
$\mathrm{O} 7$ & $\mathrm{H} 7$ & $\mathrm{O} 6$ & $2.774(3)$ & 176.9 \\
$\mathrm{O} 8$ & $\mathrm{H} 8$ & $\mathrm{O} 7$ & $2.758(3)$ & 172.6 \\
\hline
\end{tabular}




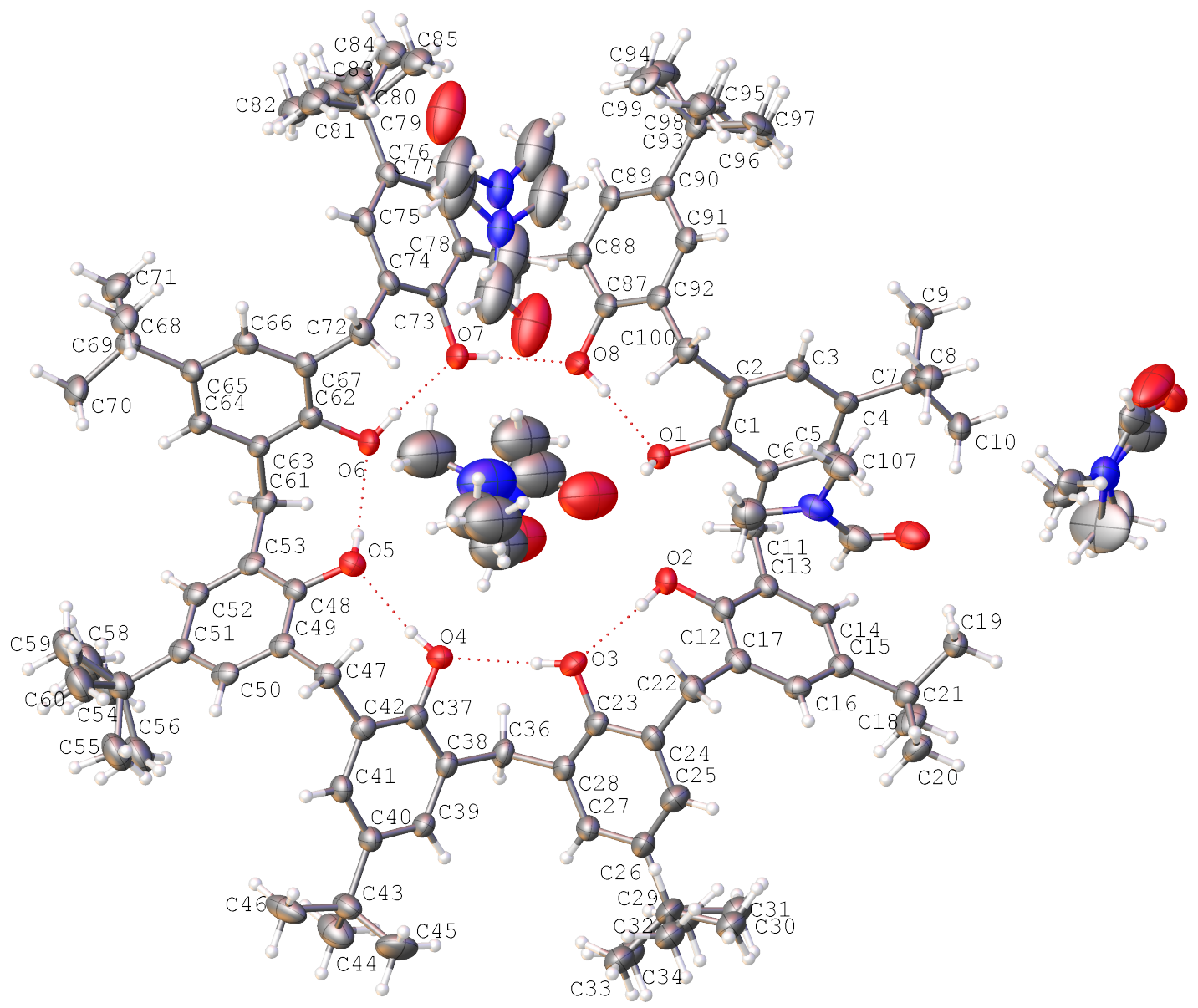

Figure S4: Labelling of molecular structure of TBC8 in 3.

Table S5: Hydrogen bonds for DMF@TBC8 (3)

\begin{tabular}{lllll}
\hline $\mathrm{D}$ & $\mathrm{H}$ & $\mathrm{A}$ & $\mathrm{d}(\mathrm{D}-\mathrm{A}) / \AA$ & $\mathrm{D}-\mathrm{H}-\mathrm{A} /{ }^{\circ}$ \\
\hline $\mathrm{O} 2$ & $\mathrm{H} 2$ & $\mathrm{O} 1$ & $2.731(6)$ & 160.5 \\
$\mathrm{O} 3$ & $\mathrm{H} 3$ & O2 & $2.725(6)$ & 162.9 \\
$\mathrm{O} 4$ & $\mathrm{H} 4$ & O5 & $2.756(6)$ & 167.4 \\
$\mathrm{O} 5$ & $\mathrm{H} 5$ & O6 & $2.718(6)$ & 143.4 \\
$\mathrm{O} 6$ & $\mathrm{H} 6$ & O7 & $2.658(6)$ & 171.7 \\
O7 & $\mathrm{H} 7$ & O8 & $2.615(6)$ & 138.3 \\
\hline
\end{tabular}




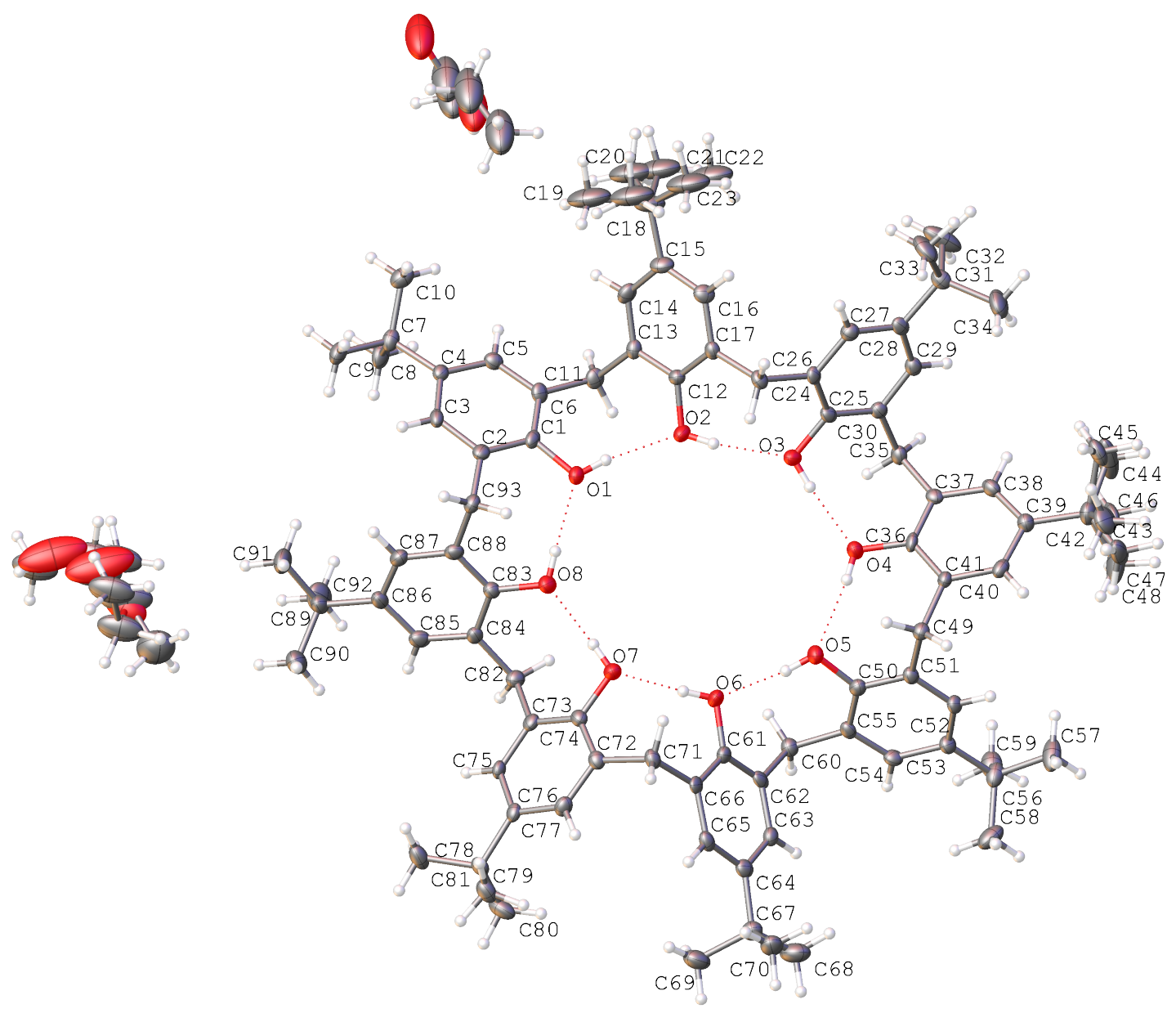

Figure S5: Labelling of molecular structure of TBC8 in 4.

Table S6: Hydrogen bonds for ethyl acetate@TBC8 (4)

\begin{tabular}{lllll}
\hline $\mathrm{D}$ & $\mathrm{H}$ & $\mathrm{A}$ & $\mathrm{d}(\mathrm{D}-\mathrm{A}) / \AA$ & $\mathrm{D}-\mathrm{H}-\mathrm{A} /{ }^{\circ}$ \\
\hline $\mathrm{O} 1$ & $\mathrm{H} 1$ & $\mathrm{O} 2$ & $2.772(4)$ & 167.8 \\
$\mathrm{O} 2$ & $\mathrm{H} 2$ & $\mathrm{O} 3$ & $2.746(4)$ & 155.4 \\
$\mathrm{O} 3$ & $\mathrm{H} 3$ & $\mathrm{O} 4$ & $2.706(4)$ & 175.0 \\
$\mathrm{O} 4$ & $\mathrm{H} 4$ & $\mathrm{O} 5$ & $2.735(4)$ & 155.4 \\
$\mathrm{O} 5$ & $\mathrm{H} 5$ & O6 & $2.635(4)$ & 165.1 \\
$\mathrm{O} 6$ & $\mathrm{H} 6$ & $\mathrm{O} 7$ & $2.606(4)$ & 175.7 \\
$\mathrm{O} 7$ & $\mathrm{H} 7$ & O8 & $2.640(4)$ & 170.7 \\
$\mathrm{O} 8$ & $\mathrm{H} 8$ & O1 & $2.729(4)$ & 171.9 \\
\hline
\end{tabular}




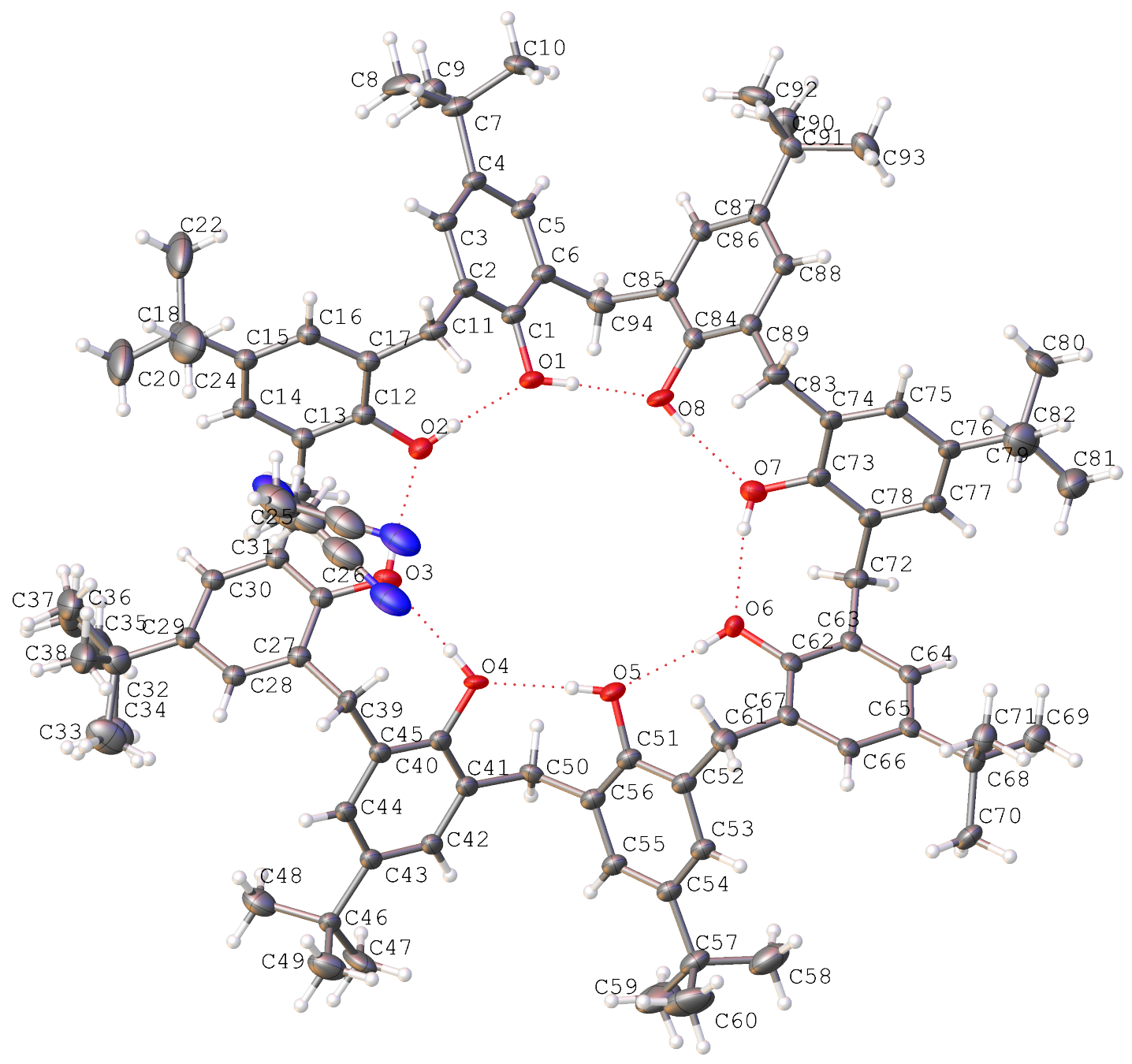

Figure S6: Labelling of molecular structure of TBC8 in 5.

Table S7: Hydrogen bonds for acetonitrile@TBC8 (5)

\begin{tabular}{lllll}
\hline $\mathrm{D}$ & $\mathrm{H}$ & $\mathrm{A}$ & $\mathrm{d}(\mathrm{D}-\mathrm{A}) / \AA$ & $\mathrm{D}-\mathrm{H}-\mathrm{A} /{ }^{\circ}$ \\
\hline $\mathrm{O} 1$ & $\mathrm{H} 1$ & $\mathrm{O} 8$ & $2.625(6)$ & 176.1 \\
$\mathrm{O} 2$ & $\mathrm{H} 2$ & $\mathrm{O} 1$ & $2.618(6)$ & 164.7 \\
$\mathrm{O} 3$ & $\mathrm{H} 3$ & $\mathrm{O} 2$ & $2.734(6)$ & 153.3 \\
$\mathrm{O} 4$ & $\mathrm{H} 4$ & $\mathrm{O} 3$ & $2.722(6)$ & 177.5 \\
$\mathrm{O} 5$ & $\mathrm{H} 5$ & $\mathrm{O} 4$ & $2.746(6)$ & 174.3 \\
$\mathrm{O} 6$ & $\mathrm{H} 6$ & $\mathrm{O} 5$ & $2.787(6)$ & 164.1 \\
$\mathrm{O} 8$ & $\mathrm{H} 8$ & $\mathrm{O} 7$ & $2.646(6)$ & 172.8 \\
\hline
\end{tabular}




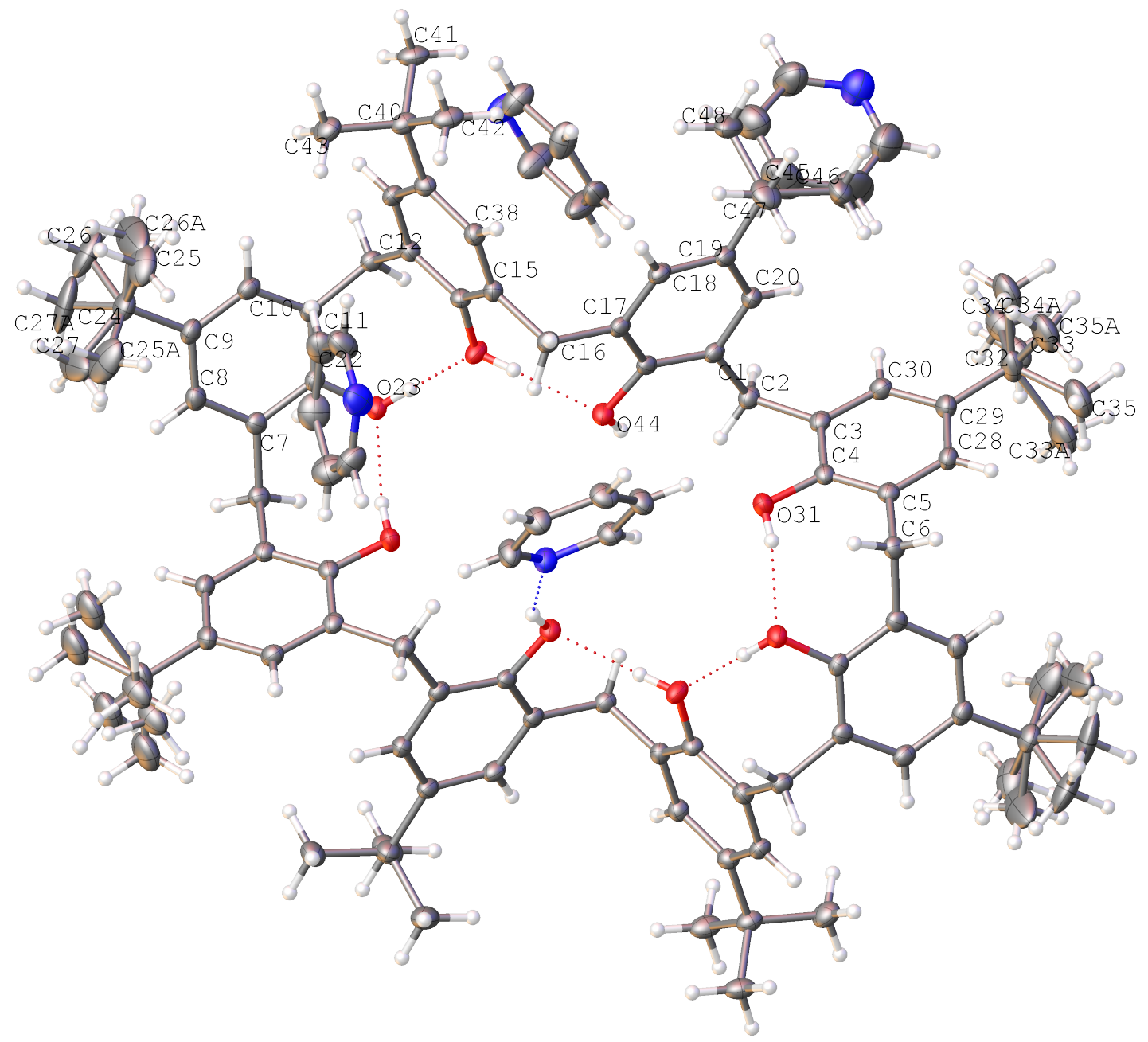

Figure S7: Labelling of molecular structure of TBC8 in 6.

Table S8: Hydrogen bonds for pyridine@TBC8 (6). ${ }^{1} 2-\mathrm{X}, 1-\mathrm{Y}, 1-\mathrm{Z}$

\begin{tabular}{lllll}
\hline $\mathrm{D}$ & $\mathrm{H}$ & $\mathrm{A}$ & $\mathrm{d}(\mathrm{D}-\mathrm{A}) / \AA$ & $\mathrm{D}-\mathrm{H}-\mathrm{A} /{ }^{\circ}$ \\
\hline $\mathrm{O} 44$ & $\mathrm{H} 44$ & $\mathrm{~N}_{4} 9^{1}$ & $2.6638(17)$ & 147.0 \\
$\mathrm{O} 39$ & $\mathrm{H} 39$ & $\mathrm{O} 44$ & $2.7462(14)$ & 170.9 \\
$\mathrm{O} 23$ & $\mathrm{H} 23$ & $\mathrm{O} 39$ & $2.7423(15)$ & 166.5 \\
$\mathrm{O} 31$ & $\mathrm{H} 31$ & $\mathrm{O}^{1} 3^{1}$ & $2.8190(15)$ & 169.5 \\
$\mathrm{C} 50$ & $\mathrm{H} 50$ & $\mathrm{O}^{2} 1$ & $3.3149(19)$ & 132.0 \\
\hline
\end{tabular}




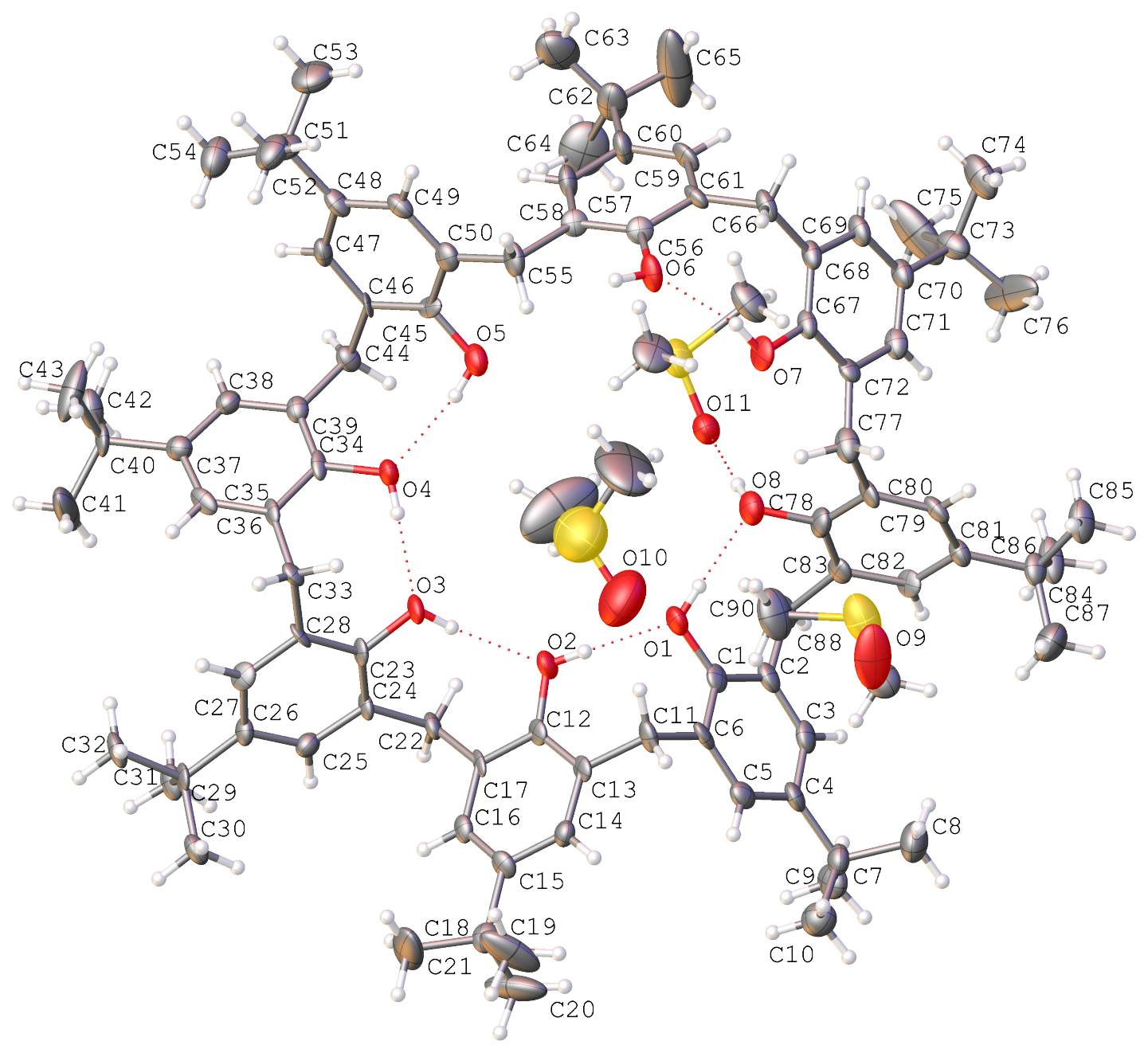

Figure S8: Labelling of molecular structure of TBC8 in 7.

Table S9: Hydrogen bonds for DMSO@TBC8 (7). ${ }^{1} 1-\mathrm{X}, 1-\mathrm{Y}, 1-\mathrm{Z}$

\begin{tabular}{lllll}
\hline $\mathrm{D}$ & $\mathrm{H}$ & $\mathrm{A}$ & $\mathrm{d}(\mathrm{D}-\mathrm{A}) / \AA$ & $\mathrm{D}-\mathrm{H}-\mathrm{A} /{ }^{\circ}$ \\
\hline $\mathrm{O} 4$ & $\mathrm{H} 4$ & $\mathrm{O} 3$ & $2.868(8)$ & 170.3 \\
$\mathrm{O} 5$ & $\mathrm{H} 5$ & $\mathrm{O} 4$ & $2.724(8)$ & 159.5 \\
$\mathrm{O} 3$ & $\mathrm{H} 3$ & $\mathrm{O} 2$ & $2.684(8)$ & 155.5 \\
$\mathrm{O} 7$ & $\mathrm{H} 7$ & $\mathrm{~S} 31$ & $3.675(7)$ & 137.5 \\
$\mathrm{O} 7$ & $\mathrm{H} 7$ & $\mathrm{O}_{1} 1^{1}$ & $2.587(10)$ & 157.3 \\
$\mathrm{O} 2$ & $\mathrm{H} 2$ & O1 & $2.646(9)$ & 164.2 \\
$\mathrm{O} 6$ & $\mathrm{H} 6$ & $\mathrm{O} 5$ & $2.797(8)$ & 162.2 \\
$\mathrm{O} 1$ & $\mathrm{H} 1$ & $\mathrm{~S} 1$ & $3.673(7)$ & 150.7 \\
$\mathrm{O} 1$ & $\mathrm{H} 1$ & O9 & $2.573(9)$ & 174.4 \\
$\mathrm{O} 8$ & $\mathrm{H} 8$ & $\mathrm{O} 7$ & $2.754(8)$ & 160.8 \\
\hline
\end{tabular}



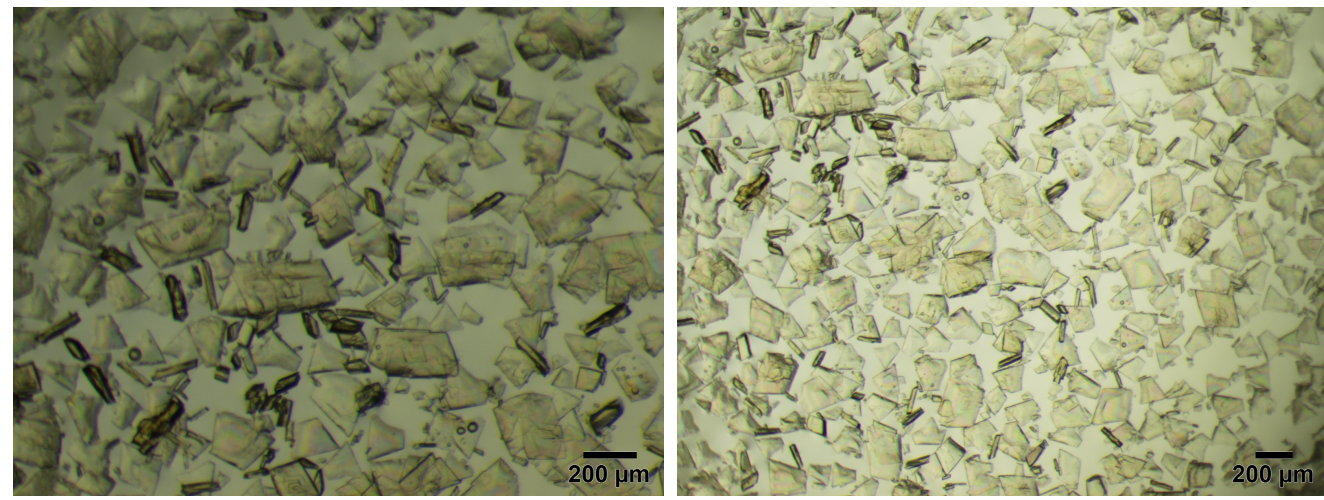

Figure S9: Photographic image of crystallization experiment of TBC8 in DMSO. Only prismatic crystal XRD data were collected. 